American Journal of Applied Sciences 6 (2): 241-246, 2009

ISSN 1546-9239

(C) 2009 Science Publications

\title{
A Short-term Management Strategy for Improving Transit Network Efficiency
}

\author{
${ }^{1}$ Amir Samimi, ${ }^{2}$ Hedayat Z. Aashtiani and ${ }^{1}$ Abolfazl (Kouros) Mohammadian \\ ${ }^{1}$ Department of Civil and Materials Engineering, University of Illinois at Chicago, \\ 842 W. Taylor Street, Chicago, IL 60607-7023, USA \\ ${ }^{2}$ Department of Civil Engineering, Sharif University of Technology, \\ P.O. Box 11365-9313, Tehran, Iran
}

\begin{abstract}
Improving the service quality of the public transport systems has always been an interesting research area in the field of transportation planning. Public transit system expansion and enhancement has been known as a recognized way to improve congested metropolitan areas. In order to make a transit system more attractive to people, both long and short term management strategies should be considered. Fleet re-assignment to a bus network, a noticeable component of the public transit system, was the focus of this study. It was introduced as a short term management strategy to help the operators maintain service quality without confusing the users by abrupt changes in the system. The proposed algorithm, Quasi-Gradient Fleet Assignment, prioritized the bus lines based on the partial derivatives of total travel time with respect to line frequencies. The algorithm improved the total system cost by $12 \%$ just by re-assigning the frequencies of the vehicles to the bus lines in a hypothetical transit network in the city of Sioux Fall, South Dakota.
\end{abstract}

Keywords: Public Transit, Bus Network, Fleet Assignment

\section{INTRODUCTION}

Public transit system, a basic part of the transportation network in urban areas, benefit society by providing mobility to people in shared vehicles. Although transit-oriented development may not always be the best way, it is one of the best solutions for transportation problems in large cities and is being focused on frequently, especially in the metropolitan areas. Public transit systems have attracted the attention of both public and private sectors for a while and have drawn lots of investments.

A significant component of a public transit system is the bus network, which has had considerable research but still needs more advanced and comprehensive methods of design, operation and maintenance. Improving the current condition of bus networks might be performed in several ways such as increasing or improving the service. Since two essential parts of the design process of an urban bus network are determining the bus routes and setting the vehicle frequencies, both bus routes and the associated frequencies might be examined to improve current operating conditions. Ideally, both fleet assignment and route selection should be modeled simultaneously. Some optimization methods have been practiced for uncongested networks ${ }^{[1]}$, however for overcrowded networks the pure optimization problems would be unmanageable and require more simplification. A common simplifying assumption is to separate the problems and approximate the optimal solution by means of heuristic algorithms. In addition to the complexity of the bus network design that presses the researchers to split it up into the fleet assignment and route selection problems, the short term nature of the fleet assignment problem requires it to be solved independent of the route selection problem. Unlike fleet assignment, route selection should be considered a long term problem. In other words, system operators should not change the bus routes very often, even if it benefits total system revenue in the short term. In general, forcing users to change their travel patterns makes them unhappy about the system and has a negative impact on their satisfaction, which leads to a decline in their long term use. On the other hand, fleet assignment could be used in short time spans as a strategy that is both economical and imperceptible to the users to avoid such inefficiencies in the system. Therefore, a reliable fleet assignment algorithm could be a helpful short term management tool for the transit network operators. The main objective of this paper is to propose a simple approach to effectively manage the fleet in a bus network.

Corresponding Author: Amir Samimi, Department of Civil and Materials Engineering, University of Illinois at Chicago, 842 West Taylor Street, Chicago, IL 60607-7023 Tel: +1(312) 996-0962 Fax: +1(312) 996-2426, 
Transit Assignment: In most of the bus network studies, a transit assignment procedure has been utilized in order to have a good representation of passengers' decision making process in a transit system. Any transit trip may be broken into four different movement types: walking, boarding, riding and alighting movements. Transit assignment predicts the behavior of all the passengers in choosing the movements, given the origin-destination (OD) demand matrix and network specifications. In order to minimize the waiting time, each traveler considers a set of attractive lines at each boarding node and the first bus that belongs to this set with at least one vacant place would be taken. Part of the complexity of the transit assignment problem arises from the stochastic nature of a passenger's waiting time, which is relative to the boarding movement. This problem has been investigated by many researchers, including Spiess and Florian ${ }^{[2]}$, DeCea and Fernandez ${ }^{[3]}$ and Babazadeh and Aashtiani ${ }^{[4]}$. In a seminal study, Spiess and Florian ${ }^{[2]}$ formulated the transit assignment problem in a linear optimization framework. The optimization problem was called Optimal Strategy and a 2-step solving algorithm was proposed for that. The algorithm finds the optimal strategy at the first step and then assigns the demand to that strategy.

DeCea and Fernandez ${ }^{[3]}$ introduced a transit assignment algorithm for congested bus networks by controlling the capacity of transit lines and stations. In this model, the passengers who are not able to take their desired bus, reroute their trip to less crowded lines in order for the model to capture the capacity restrain. Recently, Babazadeh and Aashtiani ${ }^{[4]}$ formulated the transit assignment problem in a series of complementary equations and replicated the congestion effect in the transit network perfectly. Because of the size and also nonlinearity of the complementary model, it was almost impossible to find the equilibrium solution for an extensive network. A practical solving algorithm was suggested to lessen the problem size and make it solvable for the real conditions. The algorithm consists of a bi-level decomposition pattern and a recursive route generation procedure. Nonlinearity of the model was resolved by linearizing the links' travel times. This model is able to control the transit line capacity by imposing enough penalty to the congested lines. The penalty function, shown in Equation 1, is added to all the links and is capable of keeping the flow of the link under maximum capacity by any desired accuracy.

In Equation $1, x_{a}$ and $c_{a}$ are total passenger flow and capacity in link a. $\alpha$ and $\beta$ are calculated in such a way that satisfies the continuity and differentiability of the penalty function for a given $\mathrm{p}$.

$$
\tau_{a}\left(x_{a}\right)=\left\{\begin{array}{l}
\frac{0.01}{1-\frac{x_{a}}{c_{a}}} \quad \frac{x_{a}}{c_{a}}<p \\
\alpha \frac{x_{a}}{c_{a}}-\beta \frac{x_{a}}{c_{a}} \geq p
\end{array} \quad 0<p<1\right.
$$

Bus Network Modeling: As mentioned before, one way to overcome the complexity of the bus network design is to break the problem into several independent or partially dependant sub problems. Lampkin and Saalmans ${ }^{[5]}$, Ceder and Wilson ${ }^{[6]}$, Patriksson and Labbé $^{[7]}$ are among those who focused on the route selection problem and looked into the frequency setting problem as part of that. Since route selection and fleet assignment problems are simultaneously modeled, a frequency setting algorithm may not be implemented on a transit network with predefined routes. Lampkin and Saalmans ${ }^{[5]}$ used a random search algorithm for the fleet assignment problem, which starts with an initial frequency for each bus line and then iterates and randomly tries the new frequencies from a predetermined set. Though the frequency setting procedure is not theoretically rich, this algorithm could be implemented on a prefixed set of bus lines. In some studies, frequency setting algorithm is not dissociable and could not be used for a predefined set of routes. Tom and Mohan ${ }^{[8]}$ suggested a genetic algorithm that minimizes the total cost, in order to solve the bus route choice and fleet assignment problems. In their proposed solving algorithm, the frequency of each line alters between a lower and upper bound and the transit lines with a zero frequency are automatically omitted. The remaining lines with the associated frequency build the final network. There are a few researchers who only targeted the route selection problem, such as Baaj and Mahmassani ${ }^{[9]}$. The study of Gao et al ${ }^{[10]}$ is among the few studies that only worked on the frequency setting problem. They come up with a bi-level programming model to solve the transit network design problem. Frequency setting is performed in the upper level model, while the lower level model does the transit assignment.

This study aims to propose a simple and practical algorithm to assign the fleet to a bus network and show the results in a hypothetical bus network in the city of Sioux Fall. The next section elaborates on the algorithm 
and the results will be proposed in section 3 , followed by the discussion and conclusion sections at the end.

\section{MATERIALS AND METHODS}

Fleet assignment problem could be written in the following formulation:

$$
\begin{array}{ll}
\text { Min } & \mathrm{TC}(\mathbf{f}) \\
\text { s.t. } & \mathrm{F}_{\mathrm{l}} \geq \mathrm{F}_{1}^{\min } \quad \forall \mathrm{l} \in \mathrm{L} \\
& \sum_{\mathrm{l} \in \mathrm{L}} \mathrm{F}_{1} \mathrm{~T}_{1} \leq \mathrm{M}
\end{array}
$$

$\mathrm{f}$ = Equilibrium passenger flow from transit assignment for a given set of frequency $F$ in which

$\mathrm{TC}=$ Total travel time for the users

$\mathrm{f}_{\mathrm{a}} \quad=$ Passenger flow in link $\mathrm{a}$; $\mathrm{f}$ is the vector

$\mathrm{L}=$ Set of bus lines

$\mathrm{M}=$ Total number of buses available

$F_{1}=$ Vehicle frequency of the lst line, $\mathrm{F}$ is the vector

$\mathrm{F}_{1}{ }^{\mathrm{min}}=$ Minimum permissible frequency for the lst line

$\mathrm{T}_{1} \quad=$ Cycle time for the vehicles in line 1

The model above minimizes the total users' travel time which covers the walking, boarding, in-vehicle and alighting times. The first constraint keeps the minimum permissible service. The second one controls the maximum number of running buses to be less than the available fleet size. Finally, the last constraint equilibrates the passenger flow in the network which results from the transit assignment procedure.

A recursive algorithm, called Quasi-Gradient Fleet Assignment (QGFA), is suggested in this paper to solve the model. The algorithm finds the frequency and consequently, the fleet size for each line. The method proposed by Babazadeh and Aashtiani ${ }^{[4]}$, which is capable of controlling the bus lines' capacity, is applied in this study.

As shown in Fig 1, QGFA starts with an initial solution and improves the total cost by changing the lines' frequencies. When the frequency changes are below the stopping criterion, the algorithm stops and the final frequencies will be determined. Finding an effective and logical basis to change the frequencies for the algorithm to obtain better results is the essential part. QGFA prioritizes the bus lines for absorbing more vehicles based on the partial derivatives of total travel time with respect to line frequencies. The transit lines with higher absolute value of the derivatives might have a better chance to decrease total travel time in the system. But the catchy part of the algorithm is finding the maximum interval at which the derivatives are still valid. In each iteration of the algorithm, the new frequencies will be determined by the derivatives for the current solution and also the initial allowable interval for the frequencies. Total travel time is estimated for the new frequencies in the bus network, in order to check for possible improvement in the system.

If the total travel time improves, the new frequencies will be registered; otherwise the allowable interval will be decreased.

In order to achieve a new set of lines' frequencies, as shown in Fig 1, the frequency setting problem (FSP) should be solved. In FSP, $S$ is the set of destinations; $\mathrm{R}_{\mathrm{S}}$ is the set of origins, destined for $S ; d_{r s}$ is the transit demand from $r$ to $s ; \bar{F}_{1}$ is the vehicle frequency of line 1 in the previous iteration; $U_{r s}$ is the minimum travel time from origin $r$ to destination $s$; $k$ is the iteration number; $e$ is the percentage of the allowable change for the frequencies; and $\frac{\partial \mathrm{U}_{\mathrm{rs}}^{\mathrm{k}}}{\partial \mathrm{F}_{1}^{\mathrm{k}}}$ is the partial derivatives of total travel time with respect to line frequencies. FSP is a knapsack problem and if it is solved without the first constraint, the whole fleet will be assigned to the line with the highest value of $-\sum_{\mathrm{s} \in \mathrm{S}} \sum_{\mathrm{r} \in \mathrm{R}^{\mathrm{s}}} \frac{\partial \mathrm{U}_{\mathrm{rs}}}{\partial \mathrm{F}_{\mathrm{l}}} \mathrm{d}_{\mathrm{rs}}$ which is obviously problematic. The first constraint is included in the model to solve this problem. Figure 1 shows that finding an appropriate value for $e$ makes sub-loops in the algorithm. A prerequisite step for the FSP is the derivatives estimations. A simple but computationally burdensome way of estimation is to numerically calculate the derivative values by slightly changing the frequency and running the transit assignment and calculating the change in total travel time. In order to numerically estimate the derivatives, each line's frequency is increased by $5 \%$, while the frequencies of other lines are fixed. Also $\varepsilon=0.01$ is used as the stopping criterion in this study.

The performance of the proposed algorithm is tested for a hypothetical transit system in the city of Sioux Falls, South Dakota, which is illustrated in Fig. 2. Although this bus network is hypothetical, a realistic transit network has been designed considering the actual network characteristics. Tractability of this small network with 24 nodes and 38 two-way links makes it appropriate for research purposes and makes the algorithm understandable for the readers. As shown in Table 1, almost 400,000 transit users are being served with nine bus lines every day. Each bus's capacity is 100 and the average speed is $20 \mathrm{~km} \mathrm{~h}^{-1}$ and also the walking speed is $4 \mathrm{~km} \mathrm{~h}^{-1}$. 
Am. J. Applied Sci., 6 (2): 241-246, 2009

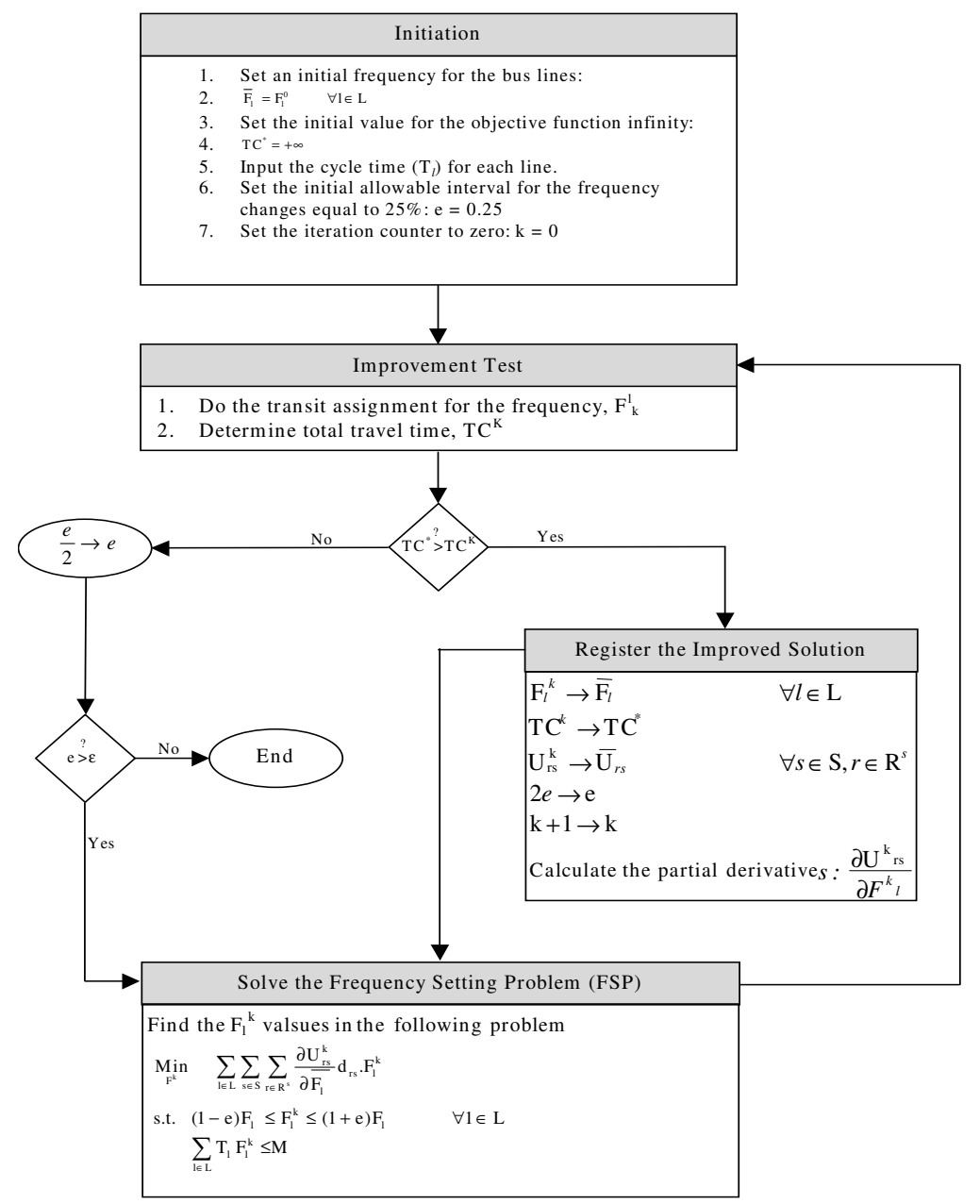

Fig. 1: Quasi-gradient fleet assignment (QGFA) flowchart

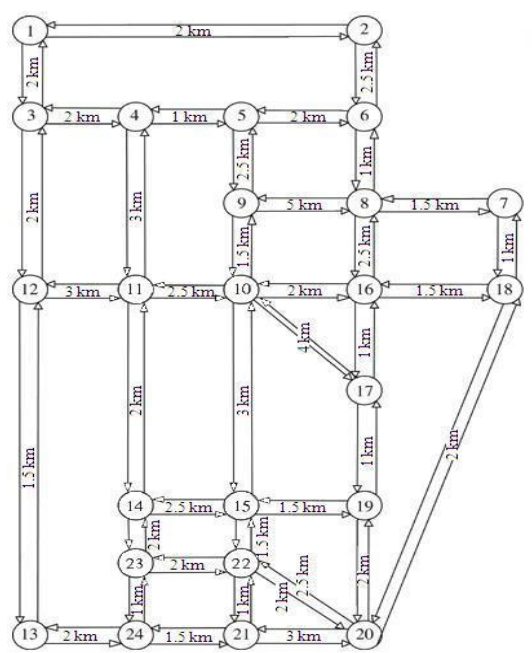

\begin{tabular}{|c|c|c|c|}
\hline Line & \begin{tabular}{|c|} 
Current \\
Fleet Size
\end{tabular} & $\begin{array}{c}\text { Cycle } \\
\text { Time (min) }\end{array}$ & $\begin{array}{c}\text { Route } \\
\text { (based on the nodes) }\end{array}$ \\
\hline 1 & 4 & 45.7 & $\begin{array}{c}4-11-10-16-17-19 \\
17-16-10-11-4\end{array}$ \\
\hline 2 & 11 & 43.3 & $\begin{array}{c}1-2-6-8-16 \\
8-6-2-1\end{array}$ \\
\hline 3 & 4 & 64.9 & $\begin{array}{c}7-8-9-10-15-22-21 \\
22-15-10-9-8-7\end{array}$ \\
\hline 4 & 10 & 45.7 & $\begin{array}{c}1-3-12-11-10 \\
11-12-3-1\end{array}$ \\
\hline 5 & 7 & 40.9 & $\begin{array}{c}4-5-9-10-16-17 \\
10-9-5-4\end{array}$ \\
\hline 6 & 2 & 40.9 & $\begin{array}{c}10-15-14-23-24 \\
23-14-15-10\end{array}$ \\
\hline 7 & 4 & 40.9 & $20-19-14-11-10-15-19-20$ \\
\hline 8 & 10 & 48.1 & $\begin{array}{c}5-4-3-12-13-24-21 \\
24-13-12-3-4-5\end{array}$ \\
\hline 9 & 2 & 28.9 & $\begin{array}{c}7-18-20-21 \\
20-18-7\end{array}$ \\
\hline
\end{tabular}

Fig. 2: Sioux Falls Bus Network 
Am. J. Applied Sci., 6 (2): 241-246, 2009

Table 1: Origin-Destination Transit Demand Matrix for the City of Sioux Falls (in 1000 Passengers/Day)

\begin{tabular}{|c|c|c|c|c|c|c|c|c|c|c|c|c|c|c|c|c|c|c|c|c|c|c|c|c|}
\hline Node & 1 & 2 & 3 & 4 & 5 & 6 & 7 & 8 & 9 & 10 & 11 & 12 & 13 & 14 & 15 & 16 & 17 & 18 & 19 & 20 & 21 & 22 & 23 & 24 \\
\hline 1 & 0 & 0.1 & 0.2 & 0.6 & 0.3 & 0.4 & 0.5 & 0.8 & 0.6 & 1.4 & 0.6 & 0.3 & 0.6 & 0.3 & 0.5 & 0.6 & 0.5 & 0.2 & 0.3 & 0.3 & 0.1 & 0.4 & 0.3 & 0.2 \\
\hline 2 & 0.1 & 0 & 0.1 & 0.3 & 0.1 & 0.5 & 0.2 & 0.5 & 0.3 & 0.6 & 0.2 & 0.2 & 0.3 & 0.1 & 0.2 & 0.4 & 0.3 & 0.1 & 0.1 & 0.2 & 0.1 & 0.2 & 0.1 & 0.1 \\
\hline 3 & 0.2 & 0.1 & 0 & 0.3 & 0.1 & 0.3 & 0.1 & 0.2 & 0.2 & 0.3 & 0.3 & 0.3 & 0.2 & 0.1 & 0.1 & 0.2 & 0.1 & 0 & 0.1 & 0.1 & 0.1 & 0.1 & 0.1 & 0.1 \\
\hline 4 & 0.6 & 0.3 & 0.3 & 0 & 0.5 & 0.5 & 0.5 & 0.7 & 0.8 & 1.2 & 1.5 & 0.7 & 0.6 & 0.5 & 0.5 & 0.8 & 0.5 & 0.1 & 0.3 & 0.4 & 0.2 & 0.4 & 0.5 & 0.3 \\
\hline 5 & 0.3 & 0.1 & 0.1 & 0.5 & 0 & 0.3 & 0.2 & 0.6 & 0.8 & 1 & 0.6 & 0.2 & 0.2 & 0.2 & 0.3 & 0.6 & 0.3 & 0.1 & 0.2 & 0.2 & 0.1 & 0.2 & 0.2 & 0.1 \\
\hline 6 & 0.4 & 0.5 & 0.3 & 0.5 & 0.3 & 0 & 0.4 & 0.8 & 0.4 & 0.8 & 0.4 & 0.3 & 0.3 & 0.2 & 0.3 & 1 & 0.6 & 0.1 & 0.3 & 0.4 & 0.1 & 0.3 & 0.2 & 0.1 \\
\hline 7 & 0.5 & 0.2 & 0.1 & 0.5 & 0.2 & 0.4 & 0 & 1.1 & 0.6 & 1.9 & 0.5 & 0.8 & 0.5 & 0.3 & 0.5 & 1.4 & 1 & 0.2 & 0.5 & 0.6 & 0.3 & 0.6 & 0.2 & 0.1 \\
\hline 8 & 0.8 & 0.5 & 0.2 & 0.7 & 0.6 & 0.8 & 1.1 & 0 & 0.9 & 1.6 & 0.9 & 0.6 & 0.6 & 0.4 & 0.7 & 2.2 & 1.4 & 0.3 & 0.7 & 0.9 & 0.4 & 0.6 & 0.4 & 0.2 \\
\hline 9 & 0.6 & 0.3 & 0.2 & 0.8 & 0.8 & 0.4 & 0.6 & 0.9 & 0 & 2.8 & 1.5 & 0.7 & 0.6 & 0.6 & 1 & 1.5 & 1 & 0.2 & 0.5 & 0.7 & 0.4 & 0.7 & 0.6 & 0.2 \\
\hline 10 & 1.4 & 0.6 & 0.3 & 1.2 & 1 & 0.8 & 1.9 & 1.6 & 2.8 & 0 & 4 & 2.1 & 1.9 & 2.2 & 4 & 4.4 & 3.9 & 0.7 & 1.8 & 2.6 & 1.3 & 2.7 & 1.8 & 0.9 \\
\hline 11 & 0.6 & 0.2 & 0.3 & 1.5 & 0.6 & 0.4 & 0.5 & 0.9 & 1.5 & 4 & 0 & 1.5 & 1 & 1.6 & 1.5 & 1.4 & 1 & 0.2 & 0.5 & 0.7 & 0.5 & 1.4 & 1.4 & 0.6 \\
\hline 12 & 0.3 & 0.2 & 0.3 & 0.7 & 0.2 & 0.3 & 0.8 & 0.6 & 0.7 & 2.1 & 1.5 & 0 & 1.4 & 0.7 & 0.8 & 0.7 & 0.7 & 0.2 & 0.3 & 0.5 & 0.4 & 0.8 & 0.7 & 0.5 \\
\hline 13 & 0.6 & 0.3 & 0.2 & 0.6 & 0.2 & 0.3 & 0.5 & 0.6 & 0.6 & 1.9 & 1 & 1.4 & 0 & 0.6 & 0.7 & 0.7 & 0.6 & 0.1 & 0.4 & 0.7 & 0.6 & 1.3 & 0.8 & 0.8 \\
\hline 14 & 0.3 & 0.1 & 0.1 & 0.5 & 0.2 & 0.2 & 0.3 & 0.4 & 0.6 & 2.2 & 1.6 & 0.7 & 0.6 & 0 & 1.3 & 0.7 & 0.7 & 0.1 & 0.4 & 0.5 & 0.4 & 1.2 & 1.1 & 0.4 \\
\hline 15 & 0.5 & 0.2 & 0.1 & 0.5 & 0.3 & 0.3 & 0.5 & 0.7 & 1 & 4 & 1.5 & 0.8 & 0.7 & 1.3 & 0 & 1.3 & 1.5 & 0.3 & 0.8 & 1.1 & 0.8 & 2.6 & 1 & 0.5 \\
\hline 16 & 0.6 & 0.4 & 0.2 & 0.8 & 0.6 & 1 & 1.4 & 2.2 & 1.5 & 4.4 & 1.4 & 0.7 & 0.7 & 0.7 & 1.3 & 0 & 2.8 & 0.5 & 1.4 & 1.7 & 0.6 & 1.2 & 0.6 & 0.3 \\
\hline 17 & 0.5 & 0.3 & 0.1 & 0.5 & 0.3 & 0.6 & 1 & 1.4 & 1 & 3.9 & 1 & 0.7 & 0.6 & 0.7 & 1.5 & 2.8 & 0 & 0.7 & 1.7 & 1.7 & 0.7 & 1.7 & 0.6 & 0.3 \\
\hline 18 & 0.2 & 0.1 & 0 & 0.1 & 0.1 & 0.1 & 0.2 & 0.3 & 0.2 & 0.7 & 0.2 & 0.2 & 0.1 & 0.1 & 0.3 & 0.5 & 0.7 & 0 & 0.4 & 0.5 & 0.1 & 0.4 & 0.1 & 0.1 \\
\hline 19 & 0.3 & 0.1 & 0.1 & 0.3 & 0.2 & 0.3 & 0.5 & 0.7 & 0.5 & 1.8 & 0.5 & 0.3 & 0.4 & 0.4 & 0.8 & 1.4 & 1.7 & 0.4 & 0 & 1.3 & 0.5 & 1.3 & 0.4 & 0.2 \\
\hline 20 & 0.3 & 0.2 & 0.1 & 0.4 & 0.2 & 0.4 & 0.6 & 0.9 & 0.7 & 2.6 & 0.7 & 0.5 & 0.7 & 0.5 & 1.1 & 1.7 & 1.7 & 0.5 & 1.3 & 0 & 1.3 & 2.5 & 0.7 & 0.5 \\
\hline 21 & 0.1 & 0.1 & 0.1 & 0.2 & 0.1 & 0.1 & 0.3 & 0.4 & 0.4 & 1.3 & 0.5 & 0.4 & 0.6 & 0.4 & 0.8 & 0.6 & 0.7 & 0.1 & 0.5 & 1.3 & 0 & 1.9 & 0.7 & 0.6 \\
\hline 22 & 0.4 & 0.2 & 0.1 & 0.4 & 0.2 & 0.3 & 0.6 & 0.6 & 0.7 & 2.7 & 1.1 & 0.8 & 1.3 & 1.2 & 2.6 & 1.2 & 1.7 & 0.4 & 1.3 & 2.5 & 1.9 & 0 & 2.2 & 1.2 \\
\hline 23 & 0.3 & 0.1 & 0.1 & 0.5 & 0.2 & 0.2 & 0.2 & 0.4 & 0.6 & 1.8 & 1.4 & 0.7 & 0.8 & 1.1 & 1 & 0.6 & 0.6 & 0.1 & 0.4 & 0.7 & 0.7 & 2.2 & 0 & 0.8 \\
\hline 24 & 0.2 & 0.1 & 0.1 & 0.3 & 0.1 & 0.1 & 0.1 & 0.2 & 0.2 & 0.9 & 0.6 & 0.5 & 0.8 & 0.4 & 0.5 & 0.3 & 0.3 & 0.1 & 0.2 & 0.5 & 0.6 & 1.2 & 0.8 & 0 \\
\hline
\end{tabular}

\section{RESULTS AND DISCUSSION}

Table 2 presents the results of the proposed fleet assignment algorithm for the abovementioned network. QGFA starts with the current fleet distribution and improves the total system cost by $12 \%$, just by reassigning the vehicles to the bus network. Having the cycle time and the line frequency, the number of vehicles for each line is calculated for the current condition. $\mathrm{p}=0.99$ and $\mathrm{e}<0.01$ are considered for the transit assignment penalty function and the stopping criterion, respectively. Table 2 shows the number of vehicles for each line, network performance parameters and some general information in the network. The other interesting point in this table is the significant decrease in the amount of walking trips in the new condition. Total walking time has dropped from almost $25 \mathrm{~h}$ to near $7 \mathrm{hrs}$, which shows a significant increase in the bus network efficiency.

Sensitivity of the algorithm to the initial solution is tested with four different scenarios. The first and second scenarios initiate with the current and uniform fleet distribution, while the third and the forth scenarios start with an extremely poor and near optimum fleet distributions, respectively.

The final fleet size for different scenarios is illustrated in Fig 3. Based on our findings, the algorithm is not able to effectively find a good solution, starting from an extremely poor fleet distribution. QGFA is a little sensitive to the initial solution; however more investigation is required for a more robust conclusion.
Table 2: Fleet Assignment Algorithm Results for Sioux Falls Network

\begin{tabular}{lll}
\hline $\begin{array}{l}\text { Parameters } \\
\text { of interest }\end{array}$ & $\begin{array}{l}\text { Initial } \\
\text { solution }\end{array}$ & $\begin{array}{l}\text { Final } \\
\text { solution }\end{array}$ \\
\hline Fleet size for line 1 & 4 & 10.2 \\
Fleet size for line 2 & 11 & 5.6 \\
Fleet size for line 3 & 4 & 11.6 \\
Fleet size for line 4 & 10 & 5.6 \\
Fleet size for line 5 & 7 & 4 \\
Fleet size for line 6 & 2 & 3 \\
Fleet size for line 7 & 4 & 6.4 \\
Fleet size for line 8 & 10 & 6 \\
Fleet size for line 9 & 2 & 1.6 \\
Total in-vehicle and waiting time (h) & 139.141 & 137.186 \\
Total walking time (h) & 25.523 & 7.543 \\
Total travel time-TC (h) & 164.664 & 144.729 \\
Average travel time for the passengers (min) & 28.392 & 23.285 \\
Number of iterations & - & 7 \\
Number of transit assignments & - & 88 \\
\hline
\end{tabular}

Number of transit assignments

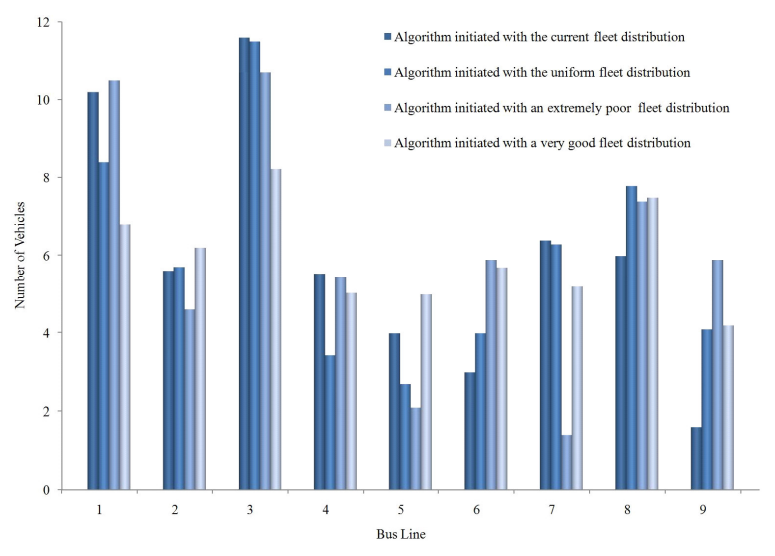

Fig. 3: Sensitivity of the algorithm to the initial solution 


\section{CONCLUSION}

Improving the service quality of the public transport systems has always been an important research area in the field of transportation planning and other related fields. Having both long and short term management strategies are a must for having a prosperous and profitable transit system. While the transit system operators need to have a semi permanent perspective for the system, short term managerial tools are also needed for them to continue providing satisfactory service to the users. Even though they have to monitor the quality of service in the system, they should not dissatisfy the customers by making perceptible changes in the systems very often. Fleet reassignment to the transit network was introduced and discussed as a short term management strategy in this paper. Mathematical formulation for the problem was elaborated and a simple but effective solving algorithm was introduced. The proposed algorithm, QuasiGradient Fleet Assignment, prioritized the bus lines based on the partial derivatives of total travel time with respect to line frequencies. The results of the algorithm were presented for a hypothetical transit networks in the city of Sioux Fall, South Dakota. QGFA was able to effectively improve the total travel time in the network by $12 \%$; however it was slightly sensitive to the initial solution. Though the algorithm should be tested in larger transit networks, it seems a reasonable and affordable short term strategy for the transit agencies to practice.

\section{ACKNOWLEDGEMENTS}

This work was partly supported by the Institute of Transportation Studies and Research at Sharif University of Technology. We would like to acknowledge Dr. Abbas Babazadeh for providing the transit assignment computer code and for his insightful comments and suggestions. Also, we are thankful to Mr. Amin Montazery and Dr. Aria Razfar for editing and formatting the manuscript.

\section{REFERENCES}

1. Fusco, G., S. Gori and M. Petrelli, 2002. A heuristic transit network design algorithm for medium size towns. Proceedings of 9th Euro Working Group on Transportation, June 10-13, 2002, Bari, Italy. pp. 652-656. $\leq$ http://www.iasi.cnr.it/ewgt/13conference/116 fus co.pdf $>$, Accessed 10 June 2008.
2. Spiess, H. and M. Florian, 1989. Optimal strategies: A new assignment model for transit networks. Transport Res. B-Meth., 23: 83-102. http://cat.inist.fr/?aModele $=$ afficheN\&cpsidt $=6736$ 982, Accessed 10 June 2008.

3. DeCea, J. and E. Fernandez, 1993. Transit assignment for congested public transport systems: An equilibrium model. Transport SCI, 27: 133-147. $\leq$ http://cat.inist.fr/?aModele= afficheN\&cpsidt $=4848485>$, Accessed 10 June 2008.

4. Babazadeh, A. and H. Z. Aashtiani, 2005. Algorithm for equilibrium transit assignment problem. Transport Res. Rec., No. 1923, Transportation Research Board of the National Academies, Washington, D.C., pp: 227-235. (doi: 10.3141/1923-24).

5. Lampkin, W. and P.D. Saalmans, 1976. The design of routes, service frequencies and schedules for a municipal bus undertaking: A case study. Oper. Res. $\quad$ Quart., $\quad$ 18: 375-393. <http://www.jstor.org/pss/3007688>, Accessed 10 June 2008.

6. Ceder, A. and N.H. Wilson, 1986. Bus network design. Transport Res. B-Meth., 20: 331-344. (doi: 10.1016/0191-2615(86)90047-0)

7. Patriksson, M. and M. Labbé, 2002. An Urban Bus Network Design Procedure. Kluwer Academic Publishers, Netherlands. $\leq \mathrm{http} / / / \mathrm{www}$. springerlink.com/content/p36r430021728248/>, Accessed 10 June 2008.

8. Tom, V.M. and S. Mohan, 2003. Transit route network design using frequency coded genetic algorithm. J. Transp. Eng. Asce., 129: 186-195. $<$ http://cat.inist.fr/?aModele $=$ afficheN\&cpsidt $=145$ 41593>, Accessed 10 June 2008.

9. Baaj, M.H. and H.S. Mahmassani, 1995. Hybrid route generation heuristic algorithm for the design of transit networks. Transport Res C-Emer., 3: 3150. (doi:10.1016/0968-090X(94) 00011-S)

10. Gao, Z., H. Sun and L.L. Shan, 2004. A continuous equilibrium network design model and algorithm for transit systems. Transport Res. B-Meth., 38: 235-250. (doi: 10.1016/S0191-2615(03)00011-0) 